\title{
Diversity of medicinal plants and their uses by the Sanger tribe of Sangihe Islands, North Sulawesi, Indonesia
}

\author{
DINGSE PANDIANGAN ${ }^{1, \boldsymbol{v}}$, MARINA SILALAHI ${ }^{2}$, FARHA DAPAS ${ }^{1}$, FEBBY KANDOU $^{1}$ \\ ${ }^{1}$ Department of Biology, Faculty of Mathematics and Natural Sciences. Universitas Sam Ratulangi. Bahu, Manado 95115, North Sulawesi, Indonesia. \\ Tel./fax.: +62-431- 863786, `email dingsepan@unsrat.ac.id, dingsepan@yahoo.com \\ ${ }^{2}$ Departement of Biology Education, Faculty of Education and Teacher Training, Universitas Kristen Indonesia. Jl. Mayjen Sutoyo No. 3, Cawang, \\ Jakarta 13510, Indonesia
}

Manuscript received: 29 October 2018. Revision accepted: 2 February 2019.

\begin{abstract}
Pandiangan D, Silalahi M, Dapas F, Kandou F. 2019. Diversity of medicinal plants and their uses by the Sanger tribe of Sangihe Islands, North Sulawesi, Indonesia. Biodiversitas 20: 611-621. The Sanger tribe is an indigenous tribe in Sangihe Islands, North Sulawesi, who still continue to use traditional medicinal plants. The objectives of this study were to document the diversity of medicinal plants and their general uses by the Sanger tribe in the selected villages of Sangihe Islands and to identify plants that have anti-cancer potential. The study was conducted with ethnobotanical approach using survey and interviews among 90 respondents selected from 9 study villages located in 3 sub-districts of Sangihe Islands. The study showed that 118 species of medicinal plants belonging to 47 families had been used by the Sanger tribe to treat 39 different types of diseases. Thirteen (13) species each were used for treating fever and ulcers, 12 species in the treatment of poisoning, 10 species each for cough and kidney diseases, 8 species for each of the diabetes mellitus and cancer or tumors, and 7 species each for itching, hypertension, physical injury and blood circulation disorders. For other diseases, the number of plant species used as medicines varied from 1 to 6 . Leaves were the most predominantly used part for medicinal purposes as this was used in case of 73 species, followed by stems (37 species), roots ( 29 species), flowers ( 25 species), fruits ( 20 species) and seeds (17 species). Twenty percent (20\% of the recorded medicinal plants were consumed directly and others after different methods of processing and preparation, such as boiling (51\%), crushing (15\%), roasting (2\%), soaking (16\%) and pasting (7\%), and a species can be used more once. A utilization of Dischidia nythesiana as medicines for cancer/tumor by the Sanger tribe has not been reported before.
\end{abstract}

Keywords: Crotalaria striata, Dischidia nythesiana, Eleutherine americana, medicinal plant diversity, Sanger tribe

\section{INTRODUCTION}

Indonesia is an archipelago with more than 17,000 islands, about 300 ethnic groups and 30,000 plant species (Indrawan et al. 2007). Plants are used for various purposes by the native tribes, mainly in their practice of traditional medicines. Research to discover medicinal properties of plants have been performed in various ways, such as by screening for biologically active secondary compounds, taxonomical studies and ethnomedicinal approach (Martin 1995) as well as through scrutiny of ancient manuscripts (Nawangningrum et al. 2004).

An ethnomedicinal approach is a research method with efficient use of the time and cost for discovering new chemical compounds for development of new medicines (Fabricant and Farnsworth 2001; Purwanto 2002), especially for chronic problems like cancer and tumors. Fabricant and Farnsworth (2001) stated that as many as $80 \%$ of drugs used globally have similar benefits with ethnomedicine. Some ethnomedicine drugs derived from quinine were adapted from indigenous knowledge of the Incas who have long used Cinchona as an anti-malarial drug (Achan et al. 2011). Moreover, legundi (Vitex trifolia) which is used as an expectorant is derived from the Javanese local wisdom (Wahyono 2008).

More ethnobotanical documentation studies are needed to intensify the research on medicinal plants, which are used by the local communities, especially since there is a tendency of high rate of erosion of local knowledge (Hoang et al. 2008). Moreover, the rate of species loss is almost parallel to the rate of loss of local knowledge (Hoang et al. 2008; Kartawinata 2010). The main objective of this research was to identify the potential medicinal plants, especially for cancers, since they are still very limited.

Cancer is the leading cause of death nowadays. Medical treatment and prevention of cancer have been carried out through removal of tumors, chemotherapy or radiotherapy (Pandiangan and Kandou 2006). Compounds that play roles in commercial cancer treatment are vincristine and vinblastine, belonging to the group of alkaloids (Alexandrova et al. 2000). They can be extracted directly from plants or produced from their precursors that are extracted from plants. Efforts to identify more plants for cancer treatment are needed. Silalahi et al. (2015b) reported that Hoya sp. and Dischidia sp. are used for cancer treatment as advertised by the medicinal herb sellers of traditional markets in Kabanjahe, North Sumatra, Indonesia.

To explore new sources of information on the use of herbs as medicines, various ethnomedicinal studies have been carried out, but they have been mostly focused on Java, Bali and some areas of Sumatra, while from other islands, such studies are very limited. Sulawesi is a unique 
island due to its biodiversity that is a combination of the western and eastern parts of Indonesia. This phenomenon has resulted in a variety of distinctive plants found only in Sulawesi, which is also the reason for the diversity of their utilization. Some studies have been attempted to inventory the medicinal plants of some regions of Sulawesi such as the Bolaang Mongondow district (Pangemanan and Rondonuwu 1991; Wardah and Danimiharja 1996; Windardi and Uji 1996; Widadeti and Roemantyo 1996; Simbala and Potabuga 2002; Bulut 2005). However, the Sanger tribe of Sangihe Island has not been studied yet in this regard.

The Sangihe Islands are a group of islands separated from the mainland Sulawesi, which is inhabited by the Sanger tribe. Documentation of medicinal plants have been carried out in Central Tabukan (Talengen, Gunung, and Tariang Baru Villages), South Tabukan (Pintareng Village) and Tahuna (Tahuna Village) by Pandiangan and Kandou (2006) and Mulalinda (2006). However, other islands in the area have not been studied yet. Kandou and Pandiangan (2006) found that medicinal plants rich in alkaloids, such as Catharanthus roseus, Carica papaya, Coleus scutellarioides (mayana), Coleus amboinicus (panise), Codiaeum variegatum (kalubahangi), Ficus emery (hope), Melaleuca bracteata (balakama), Peperomia pellucida (suruhan), Piper betel, Cassitha filiformis (taliputri), Morinda citrifolia (seha) and Persea gratissima were found here. Therefore, this study was undertaken with the objectives of determining the diversity of medicinal plants and their use by the Sanger tribe in the Sangihe Islands in general and identifying the medicinal plants which are used in cancer treatment, in particular.

\section{MATERIALS AND METHODS}

\section{Study area}

The study was conducted from January to July 2017, in nine villages located in Sangihe Islands District and Sitaro (Siau Tagulandang Biaro) Islands District, North Sulawesi Province, Indonesia (Figure 1). The Sangihe Islands consist of 105 islands of which 79 islands are uninhabited and 26 islands are inhabited, while Sitaro Islands consist of 47 islands of which 37 islands are uninhabited and 10 islands are inhabited by the Sanger ethnic group. The Sangihe Islands are geographically located between $2^{\circ} 43^{\prime \prime}-4^{\circ}$ $44^{\prime} 22^{\prime \prime} \mathrm{N}$ and $125^{\circ} 9^{\prime} 28^{\prime \prime}-125^{\circ} 56^{\prime} 57^{\prime \prime} \mathrm{E}$; while Sitaro Islands are located between $2^{\circ} 4^{\prime} 13^{\prime \prime}-2^{\circ} 52^{\prime} 47^{\prime \prime} \mathrm{N}$ and $125^{\circ} 9^{\prime} 28^{\prime \prime}$ $125^{\circ} 24^{\prime} 25^{\prime \prime}$ E (BPS 2017, 2018). The villages selected for the present study were Lesa, Tapuang, and Batulewehe of East Tahuna sub-district of Sangihe Islands District; Mala, Naha, and Kalakube of North Tabukan sub-district of Sangihe Islands District; and Lumbo, Bawoleu, and Bawo of North Tagulandang sub-district of Sitaro Islands District.

\section{Sanger tribe}

Majority of people belonging to Sanger tribe are fishermen, living in the coastal areas, and farmers who live in the mountain areas. The farmers mainly cultivate coconut (Cocos nucifera), nutmeg (Myristica fragrans) and clove (Syzygium aromaticum) (Figure 2).

\section{Data collection}

Data was collected through ethnobotanical approach of semi-structured, in-depth interviews and participatory observations. A group of 90 respondents, which consisted of 10 people from each of the study village, were interviewed. The respondents were religious leaders, staff of the village administrative office, respected elders of the village and traditional healers who were selected using purposive snowball sampling method. Herbarium specimens of medicinal plants were collected and their local names, parts used and method of utilization was noted along with uses.

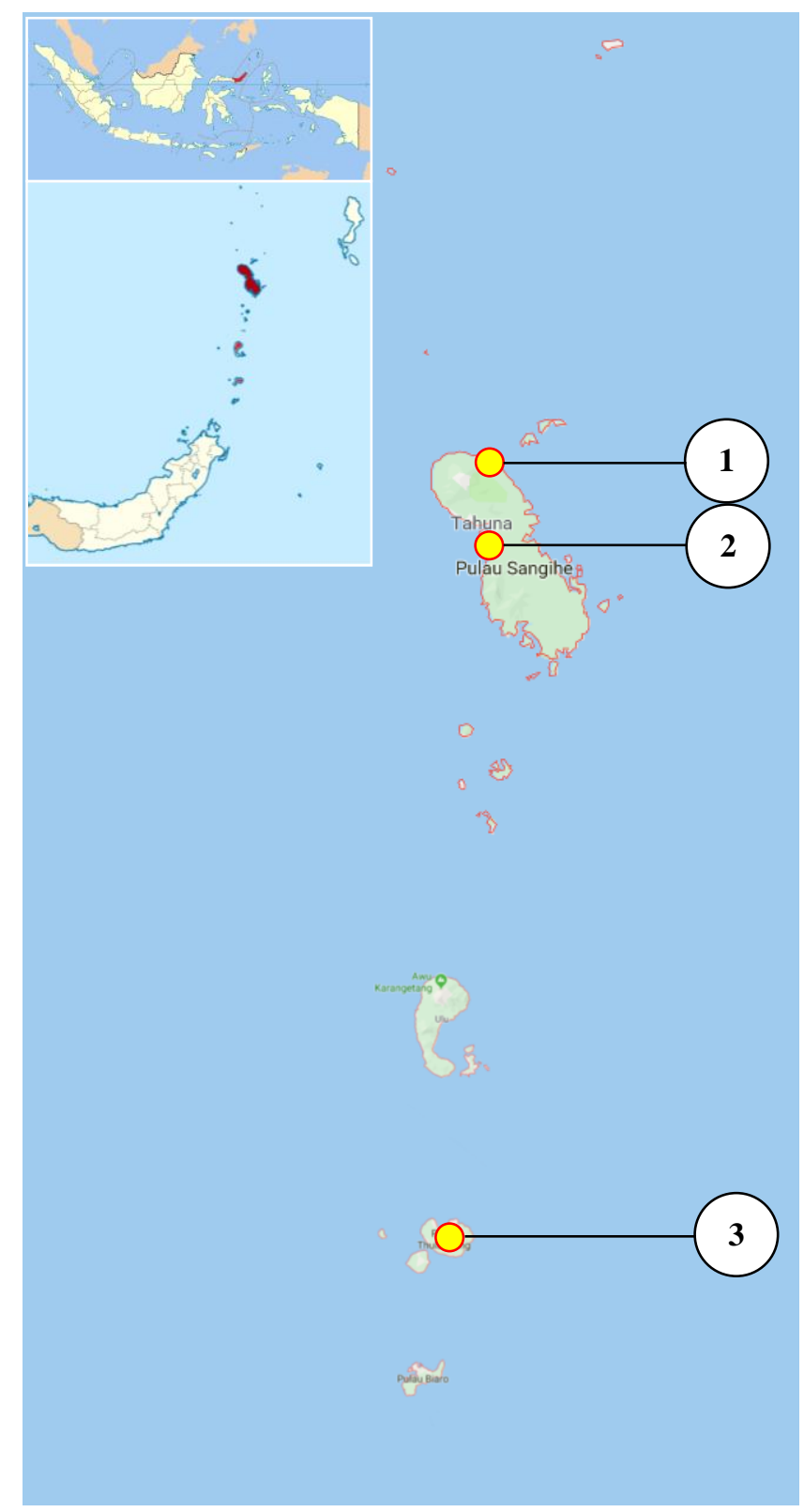

Figure 1. The research sites in the North Tabukan (1) and East Tahuna (2) sub-districts of Sangihe Islands District and North Tagulandang (3), Sitaro Islands District, North Sulawesi Province, Indonesia 

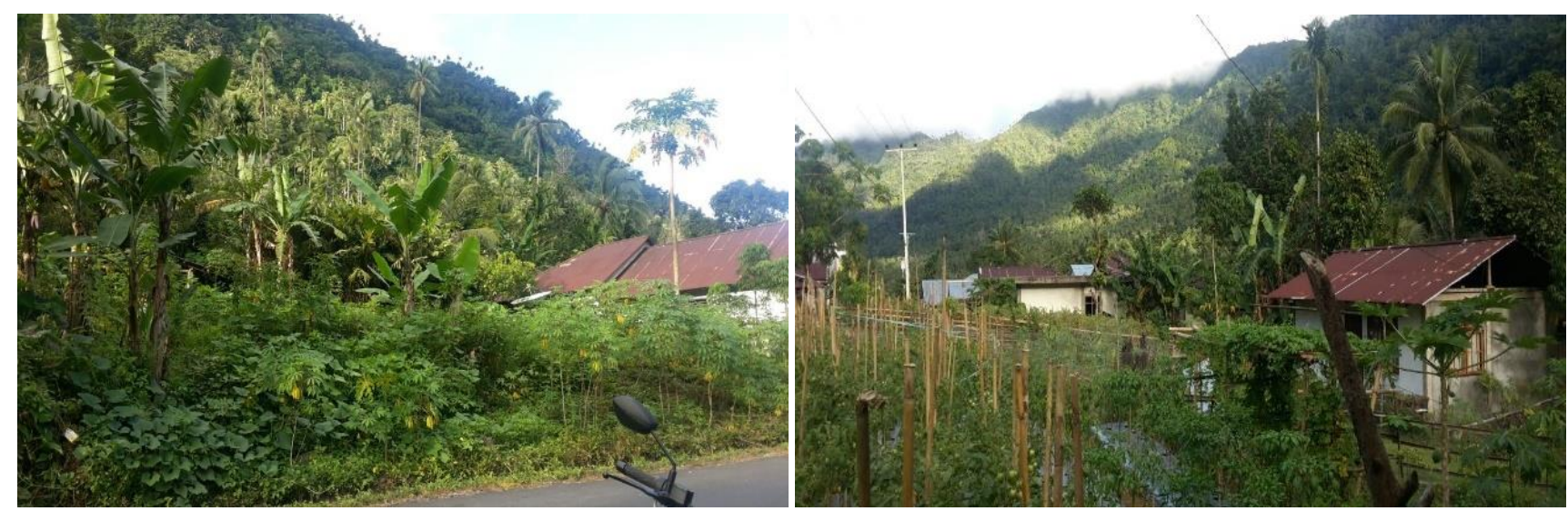

Figure 2. Research sites in Lumbo Village, North Sulawesi, Indonesia showing coconut cultivation in coastal lowland area (left), and cultivation of nutmeg in mountainous area (right)

Interviews were based on modified methods of Martin (1995), Alexiades and Sheldon (1996) and Kandou and Pandiangan (2006). Medicinal plants collected from the field were identified refer to Backer and Bakhuizen v.d. Brink (1963-1968). The botanical names of the plants were then verified using the online www.theplantlist.org database version 2017.

\section{Data analysis}

The ethnomedicinal data collected in this study were analyzed qualitatively and quantitatively. The index of similarity and inequality was calculated using Jaccard Index (Ji) equation (Mueller-Dumbois and Ellenberg 1974; Krebs 1978;).

Jaccard index ( $\mathrm{Ji})$ was calculated using the equation:

$$
\mathrm{Ji}=\frac{a}{a+b+c}
$$

Where:

a: Number of species found in villages A and B

$\mathrm{b}$ : Number of species found in village $\mathrm{A}$, but not found in village $B$

c: Number of species found in village $B$, but not found in village $A$

\section{RESULTS AND DISCUSSION}

\section{Medicinal plants used by the Sanger tribe}

The Sanger tribe was found to be using a total of 118 species of plants as medicinal, which belongs to 46 families. Table 1 provides family-wise list of all these plants with information on their local names, scientific names, uses, habit and location or villages where the plants were found. The number of species of medicinal plants recorded in this study was lower than that of the Batak Simalungun tribe (Silalahi et al. 2015a) and Batak Karo tribe (Purba et al. 2016), but higher than that of the Lahat tribe (Harmida and Yuni 2010). The number of species recorded during ethnobotanical studies is strongly influenced by the natural plant diversity of the study area as well as the number of respondents. Anwar et al. (1984) stated that Sumatra has a very high floral diversity, with about 10,000 species of plants, while the Sangihe Islands are floristically less diverse. The species diversity within the area is directly proportional to the total area of the islands (Cox and Moore 2008).

Plant family-wise analysis of medicinal plants indicated that Lamiaceae (11 species), Euphorbiaceae (9 species), Acanthaceae (7 species), Zingiberaceae (6 species), Malvaceae ( 5 species) and Verbenaceae $(5$ species) were the families with largest number of species found in the study. Lamiaceae members were the most often found plants since they do not require any special maintenance, are resistant to deficiency and excess of water, and are easily propagated through cuttings and seeds. Three species collected in the present study were identified only to the genus level. They are tate filipina (Coleus sp.2), panesse (Coleus sp.1), and betadin (Sterculia sp.). The plant called tate filipina (Coleus sp.2) has been known as a cure for cancer. The limitations in collecting the plant specimens were the major constraints for further identification of these unidentified species.

Members of Acanthaceae have been used to treat various diseases, such as increasing the number of blood cells (Asystasia gangetica, Hemigraphis colorata), fever (Graptophilum pictum), malarial fever (Andrographis paniculata), kidney disease (Strobilanthes crispus), and toothache (Barleria prionitis). Lamiaceae members are used for treating fever ( $C$. amboinicus), cough $(C$. scutellaroides) and heart disease (Pogostemon hedgei). Moreover, there are three species of the Euphorbiaceae family that are known to treat cases of poisoning: Jatropha curcas, Phyllanthus niruri and Euphorbia antiquorum.

Some of the medicinal plants found useful in this study have already been reported to be medicinal by earlier studies and they mainly belong to Zingiberaceae family, such as Curcuma longa, Alpinia galanga, Zingiber officinale and, Curcuma xanthorrhiza (Silalahi et al. 2015a,b). However, medicinal uses of species, such as Clerodendrum sahelangi, Leucas lavandulifolia, 
Clerodendrum calamitosum, Talinum triangulare, Abelmoschus manihot, Eleutherine americana, Coleus sp., Pedilanthus tithymaloides, Dischidia nythesiana, Eurycles amboinensis and Barleria prionitis are new since they have not been reported before.

Analysis of number of medicinal plants employed to treat various diseases showed that a maximum number of 13 species each were used to treat fever and ulcers. Other major diseases were poisoning (12 species), cough (10 species), kidney disorders (10 species), diabetes (8 species), cancer/tumors (8 species), itching (7 species), hypertension ( 7 species), injuries or cuts ( 7 species) and circulation disorders ( 7 species). Medicinal plants were also used to treat about 28 other diseases and the number of species used against each such disease varies from one to six (Figure 3).

The plants that were found to be used to treat fever were Hemigraphis colorata, Pluchea indica, Euphorbia thymifolia, J. curcas, C. amboinicus, Oxalis corniculata, and Piper betel. The utilization of Piper betel and J. curcas to treat fever has also been reported from the Batak Simalungun (Silalahi et al. 2015a), Karo (Purba et al. 2016) and Minang (Khairiah 2017) tribes. There is a belief among the villagers that certain plants are 'cold' in nature, thus they can be used to treat fever and lower the patient's body temperature.

Silalahi et al. (2015a) stated that the Simalungun ethnic group use plants that have coarse leaves to treat kidney disorders. The Sanger tribe uses Orthosiphon spicatus and $S$. crispus to treat kidney disorders. They are both known as diuretics and the leaf of Orthosiphon spicatus has also been listed as diuretic (MoH 1977). The local people also exchange information on the medicinal uses of plants with other communities.

The local use of Artemisia vulgaris, Momordica charantia, P. niruri, J. curcas to treat cases of poisoning may be considered as relatively new reports. $P$. niruri has been known as a diuretic $J$. curcas is known to cure fever and $M$. charantia has been reported to be used in the treatment of diabetes (Wijayakusuma 1996a, 1996b, 1996c, 2000). The Sanger ethnic group has been using about 12 species to treat cases of poisoning, such as bites of snakes, insects and other animals. For medicinal purposes, the plants are consumed fresh or as a simple extract in water. Parts of the plants that are believed to be having medicinal properties are usually boiled and then consumed with added palm sugar.

A total of eight species of medicinal plants were used in cancer or tumor treatment. They are D. nythesiana, A. vulgaris, Crotalaria striata, E. americana, Catharanthus roseus, Philippines Coleus sp. 1., Derris elliptica and Coleus sp. 2. Catharanthus roseus has long been used for cancer treatment because it produces the alkaloid vincristine and vinblastine, the compounds that have been shown to inhibit the growth of cancer cells. Thus, this plant, which originated from Madagascar, has been commercialized as a medicine for cancer. D. nythesiana, A. vulgaris, $C$. striata, and $E$. americana have also been known as cancer drugs.

As many as 79 species of the medicinal plants used by the Sanger tribe have been cultivated, while the remaining 39 species were found in the wild. The wild plants can be found in roadsides, in gardens growing wild and also in the forest. The medicinal plants that are often cultivated by the locals are those with additional benefits, such as spices $(C$. longa, A. galanga, Z. officinale), hedgerow (J. curcas, $S$. crispus), vegetables (C. amboinicus, Allium cepa), and fruit trees (Carica papaya, Annona muricata).

Different parts of the plants are utilized for medicinal purposes. The parts used in current study are the roots (29 species), stems (37 species), leaves (73 species), flowers (25 species), fruits (20 species) and seeds (17 species) (Fig. 4). Among all parts of a plant, medicinal property is mainly found in the leaves $(36 \%)$, followed by stems $(18 \%)$, roots (14), flowers (13\%), fruits (10\%) and seeds (9\%).

The methods of preparation of the medicinal plants were boiling in water $(51 \%)$, crushing $(15 \%)$, direct consumption $(20 \%)$, roasting $(2 \%)$, soaking $(16 \%)$ and pasting $(7 \%)$. Medicinal plants that are consumed directly can be done by eating plant parts or parts of the extract. One type of plant can be used in more ways, this results in a total plant of more than $100 \%$. For example, the Piper betle, $C$. longa can be used directly, that is by taking fresh extracts or by stirring first. Boiling, as a method of preparation, is seen to be more hygienic and simpler for consumers. Moreover, boiling in water helps to lower the level of bitterness, and the secondary metabolites present in the plants get dissolved in water (Harbone 1987). Thus, boiling results in the extraction of the active compounds and, therefore, it increases the efficacy of the medicinal preparations.

\section{Potential anti-cancer plants}

The Sanger tribe uses a total of eight species of medicinal plants for the treatment of cancer, or tumor $D$. nythesiana is a new report. D. nythesiana, A. vulgaris, $C$. striata, E. americana and Coleus sp. that belong to four different families. The Sanger tribe believes that cancer or tumor is difficult to cure and they often result in death. Local people recognize cancer with symptoms of large lumps in the body, and cause interference with all parts of the body. Treatment for cancer is done by taking various Dischidia herbs for a long time. Alkaloid from Catharanthus roseus, such as vincristine and vinblastine, as well as taxol from Taxus sp. are commercially available cancer drugs that are extracted from plants (Munim and Hanani 2011). Tests on anti-cancer activity are mostly done in vitro using cell line. 
Table 1. Family-wise list of medicinal plants utilized by the Sanger tribe in Sangihe Islands and Sitaro Islands districts, North Sulawesi Province, Indonesia. For each plant, local name/s, scientific name, uses, name of the village (s), where used and life form is given

\begin{tabular}{|c|c|c|c|c|}
\hline Scientific name & Local name & Uses & Villages & Lifeform \\
\hline \multicolumn{5}{|l|}{ Acanthaceae } \\
\hline Andrographis paniculata (Burm.f.) Nees & Sambiloto & Malaria fever, Liver problems & TG, BE, LA, BO & Herb \\
\hline Asystasia gangetica And. & Bungang hisa & Anemia & LO, BU & Shrub \\
\hline Barleria prionitis $\mathrm{L}$. & Kalu burung & Scabies, itching, toothache & TG, LA, NA & Shrub \\
\hline Graptophyllum pictum $\mathrm{L}$. & Limpadaleng & Ulcer & NA, LO & Shrub \\
\hline Hemigraphis colorata $\mathrm{L}$. & Marilah & Cough, bleeding, fever, anemia & TG, BE, LA, MA, LO, BU & Herb \\
\hline Justicia gendarussa Burm. f. & Nggahusa & Gout, cholera & TG, NA, MA, LO, BU, BO & Shrub \\
\hline Strobilanthes crispus Blume & Kaca beling & Kidney disease & TG, BE, LA, NA, LO, BO & Shrub \\
\hline \multicolumn{5}{|l|}{ Alliaceae } \\
\hline Allium cepa $\mathrm{L}$. & Lansuna mahamu & Ulcer & LA, LO, BU & Herb \\
\hline Allium schoenoprasum $\mathrm{L}$. & Ganda & Hypertension, diarrhea, dysentry & $\begin{array}{l}\text { TG, BE, LA, NA, KE, MA, } \\
\text { LO, BU, BO }\end{array}$ & Herb \\
\hline \multicolumn{5}{|l|}{ Amaranthaceae } \\
\hline Amarantus spinosus L. & Bayam putih & Ulcer & NA, KE, BU & Herb \\
\hline Celosia argentea $\mathrm{L}$. & Kaliapa umboh & Anemia & TG, LA, NA & Herb \\
\hline Celosia cristata $\mathrm{L}$. & Sasihungu manu & Measles & LA, NA & Herb \\
\hline \multicolumn{5}{|l|}{ Amaryllidaceae } \\
\hline Eurylesamboinensis Loud & Walantuluhi & Ulcer & TG, BO & Herb \\
\hline \multicolumn{5}{|l|}{ Annonaceae } \\
\hline Annona muricata $\mathrm{L}$. & Nangka walanda & $\begin{array}{l}\text { Gout, stomach ache, heart } \\
\text { disease, headache }\end{array}$ & LA, NA, MA, BO & Tree \\
\hline \multicolumn{5}{|l|}{ Apiaceae } \\
\hline Centella asiatica $\mathrm{L}$. Urban & Dalimase & Kidney disease, Chicken fox & $\begin{array}{l}\text { TG, BE, KE, MA, LO, BU, } \\
\text { BO }\end{array}$ & Herb \\
\hline Foeniculum vulgare Mill & Anis & Cough & $\mathrm{BO}$ & Herb \\
\hline \multicolumn{5}{|l|}{ Apocynaceae } \\
\hline Alstonia scholaris $\mathrm{R} . \mathrm{Br}$ & Galiti & Malaria fever, headache & $\mathrm{BO}$ & Tree \\
\hline Catharanthus roseus (L.) G. Don. & Gendola & $\begin{array}{l}\text { Diabetes mellitus, cancer, } \\
\text { hypertension }\end{array}$ & $\begin{array}{l}\text { LA, NA, KE, MA, LO, BU, } \\
\text { BO }\end{array}$ & Herb \\
\hline Plumeria acuminata Ait & Kamboja & Ulcer & $\mathrm{BE}$ & Tree \\
\hline \multicolumn{5}{|l|}{ Araceae } \\
\hline Homalomena occulta (Lour.) Schott. & Kalintama & Stroke & $\mathrm{BU}$ & Herb \\
\hline \multicolumn{5}{|l|}{ Arecaceae } \\
\hline Areca catechu $\mathrm{L}$. & Tilade & $\begin{array}{l}\text { Diabetes mellitus, diarrhea, } \\
\text { disentry }\end{array}$ & TG, LA, NA, KE, BU & Tree \\
\hline Arenga pinata (Wurmb) Merr. & Pohon seho & Kidney disease & $\mathrm{LO}, \mathrm{BU}$ & Tree \\
\hline Cocos nucifera $\mathrm{L}$. & Wango & Measles, influenza & NA, LO, BU, BO & Tree \\
\hline \multicolumn{5}{|l|}{ Araliaceae } \\
\hline Nothopanax scutellarium Merr & Daun mangkok & Hair loss & $\mathrm{BE}$ & Herb \\
\hline Panax ginseng C.A. Mey & Ginseng & Aphrodisiac & LO & Herb \\
\hline \multicolumn{5}{|l|}{ Asclepiadaceae } \\
\hline Dischidia nythesiana & Lintakuhi & Cancer & $\mathrm{LO}, \mathrm{BO}$ & Herb \\
\hline \multicolumn{5}{|l|}{ Asteraceae } \\
\hline Artemisia vulgaris L. & Daun tatutup & Heart disease, poisoning, cancer & TG, NA, KE, BO & Herb \\
\hline Gynura sp. & Daun berkat & Diabetes mellitus & $\mathrm{TG}, \mathrm{BU}, \mathrm{BO}$ & Herb \\
\hline Pluchea indica $\mathrm{L}$. & Balontas & Fever & $\mathrm{LO}, \mathrm{BO}$ & Herb \\
\hline Spilanthes ocymifolia Moore & Ruku sinsing & Diabetes mellitus, toothache & LO & Herb \\
\hline \multicolumn{5}{|l|}{ Balsaminaceae } \\
\hline Impatiens balsamina $\mathrm{L}$. & Laka & Injury, disentry & TG, LA, NA, MA, BU & Herb \\
\hline \multicolumn{5}{|l|}{ Bromeliaceae } \\
\hline Annanas comosus L. (Merr.) & Nanasi & Kidney disease & $\mathrm{KE}, \mathrm{LO}, \mathrm{BU}$ & Herb \\
\hline \multicolumn{5}{|l|}{ Caesalpiniaceae } \\
\hline & \multicolumn{3}{|c|}{ Caricaceae } & Herb \\
\hline Carica papaya $\mathrm{L}$. & Kapala & Hypertension, worm disease & MA, LO, BU & Herb \\
\hline \multicolumn{5}{|l|}{ Commelinaceae } \\
\hline Rhoe discolor (L. Her) Hance & Adam dan hawa & Hypertension & TG, BE, LA, LO, BU, BO & Herb \\
\hline Convolvulaceae & & & & \\
\hline Ipomoea pes-caprae (L.) Sweet & Batata pante & Sprain & $\mathrm{BO}$ & Herb \\
\hline Cucurbitaceae & & & & \\
\hline $\begin{array}{l}\text { Momordica charantia } \mathrm{L} \text {. } \\
\text { Crassulaceae }\end{array}$ & Papare & Poisoning, body odor & TG, NA & Herb \\
\hline
\end{tabular}




\begin{tabular}{|c|c|c|c|c|}
\hline Kalanchoe pinnata (Lamp.) Pers & Tahulending & Ulcer, fever & TG, BE, LA, LO, BO & Herb \\
\hline \multicolumn{5}{|l|}{ Euphorbiaceae } \\
\hline Euphorbia antiquorum $\mathrm{L}$. & Kau leper & Poisoning & $\mathrm{BE}, \mathrm{LO}, \mathrm{BO}$ & Herb \\
\hline Euphorbia hirta L. & Ruku tinta & Cataract & TG, LA, KE, LO BO & Herb \\
\hline Euphorbia thymifolia L. & Onasi & Fever & $\mathrm{KE}, \mathrm{BU}$ & Herb \\
\hline Euphorbia tirucalli L. & Kau duhi & Fracture & TG, BU & Herb \\
\hline Jatropha curcas L. & Talunsina & $\begin{array}{l}\text { Fever, itching, kidney disease, } \\
\text { gastrointestinal disorder, fever, } \\
\text { poison disease }\end{array}$ & $\begin{array}{l}\text { TG, BE, LA, NA, KE, MA, } \\
\text { LO, BU, BO }\end{array}$ & Herb \\
\hline Jatropha gossypifolia $\mathrm{L}$. & $\begin{array}{l}\text { Talunsia } \\
\text { mahamu }\end{array}$ & Typhoid fever, Childbirth & NA, LO & Shrub \\
\hline Pedilanthus tithymaloides (L.) Poit & Bungang perak & Venomous bites & TG, LO & Herb \\
\hline Phyllanthus niruri L. & Dukung anak & Kidney disease, poison disease & TG, LA, KE & Herb \\
\hline Sauropus androginus Merr & Katu & Increase breast milk & LA, NA, MA, LO & Shrub \\
\hline \multicolumn{5}{|l|}{ Lamiaceae } \\
\hline Coleus amboinicus Lour & Tabale & Fever, fracture & $\mathrm{BE}$ & Herb \\
\hline Coleus blumei Benth & Mahampusiram & Internal disease & $\mathrm{BL}, \mathrm{BO}$ & Herb \\
\hline Coleus scutellarioides (L.) Benth. & Titate & Cough, poisoning & TG, NA, KE, LO, BU, BO & Herb \\
\hline Coleus sp.1 & Panesse & Cough & TG, BE, LA, LO, BU, BO & Herb \\
\hline Coleus sp. 2 & Tate filipina & Cancer & TG & Herb \\
\hline Leucalavanduli folia Smith & Haerani & Nose bleeding & $\mathrm{LA}, \mathrm{BO}$ & Herb \\
\hline Mentha crispa Rumph & Salasi & Gastrointestinal disorder & NA & Herb \\
\hline Ocimum basilicum L. & Balakama & Scabies, headache & TG, LA, NA, KE & Herb \\
\hline Orthosiphon spicatus B.B.S & Kumis kucing & Kidney disease, poisoning & TG, BE, NA, LO, BO & Herb \\
\hline Pogostemon cablin (Blanco) Benth. & Mahangkirang & Hair care & $\mathrm{TG}, \mathrm{LO}, \mathrm{BU}, \mathrm{BO}$ & Herb \\
\hline $\begin{array}{l}\text { Pogostemon hedgei V.S.Kumar \& } \\
\text { B.D.Sharma }\end{array}$ & Lihunu & Stomach ache & $\mathrm{BU}, \mathrm{BO}$ & Herb \\
\hline \multicolumn{5}{|l|}{ Lauraceae } \\
\hline Lawsonia inermis $\mathrm{L}$ & Lakasiang & Internal disease & $\mathrm{BE}, \mathrm{LA}$ & Shrub \\
\hline Persea americana Mill & Alpokat & Hypertension & $\begin{array}{l}\text { TG, BE, LA, NA, KE, MA, } \\
\text { LO, BU, BO }\end{array}$ & Tree \\
\hline \multicolumn{5}{|l|}{ Liliaceae } \\
\hline Aloe vera $\mathrm{L}$. & Lidah buaya & Diabetes mellitus, cough, hair care & TG, LA, NA, BU, BO & Herb \\
\hline Coryline fruticosa (L.) A Chev. & Bungang & Injury & $\mathrm{KE}$ & Shrub \\
\hline $\begin{array}{l}\text { Eleutherine americana Merr. } \\
\text { Malvaceae }\end{array}$ & Bawang babi & Cancer & TG & Herb \\
\hline Abelmoschus manihot Pohl. & Saroro & $\begin{array}{l}\text { Diarrhea, appendix disolder, } \\
\text { delivery }\end{array}$ & $\begin{array}{l}\text { TG, BE, LA, NA, KE, MA, } \\
\text { LO, BU, BO }\end{array}$ & Shrub \\
\hline Gossypium herbaceum L. & Kapesi & Cough & LA, KE, MA, BO & Shrub \\
\hline Hibiscus rosa-sinensis $\mathrm{L}$. & Hambuanga & Ulcer & BE, LA, KE, MA, BU & Shrub \\
\hline Hibiscus sp. & Rosi ambon & Cancer & LO & Tree \\
\hline Sida rhombifolia $\mathrm{L}$. & Rumpu sosapu & Tonsil, Rheumatism & TG, BU, BO & Shrub \\
\hline \multicolumn{5}{|l|}{ Menispermaceae } \\
\hline Tinospora crispa $\mathrm{L}$. & Kapu wanehi & Diabetes mellitus & BU & Herb \\
\hline Tinospora tuberculata Blume & Kapu ambong & Malaria fever, heart disease & $\mathrm{LO}, \mathrm{BU}, \mathrm{BO}$ & Herb \\
\hline \multicolumn{5}{|l|}{ Mimosaceae } \\
\hline Leucaena glauca Benth & Marawelesi & Scabies & $\mathrm{BE}, \mathrm{BU}$ & Tree \\
\hline Mimosa pudica $\mathrm{L}$. & Putri malu & Cough & $\mathrm{BU}$ & Herb \\
\hline \multicolumn{5}{|l|}{ Moringaceae } \\
\hline Moringa oleifera Lam. & Kelor & Diabetes mellitus & LA & Tree \\
\hline \multicolumn{5}{|l|}{ Musaceae } \\
\hline Musa paradisiaca $\mathrm{L}$. & Wusa manduru & Heart disease & $\mathrm{BE}, \mathrm{LA}$ & Herb \\
\hline Musa textilis Nee & Wusa hote & Kidney disease & $\mathrm{BE}$ & Herb \\
\hline \multicolumn{5}{|l|}{ Myrtaceae } \\
\hline Eugenia aquea Burm. f & Kapeta mahamu & Microba infection in mouth & TG, KE & Tree \\
\hline Psidium guajava $\mathrm{L}$ & Goyawas & Diarrhea, stomach ahce & $\begin{array}{l}\text { TG, BE, LA, NA, KE, MA, } \\
\text { LO, BU, BO }\end{array}$ & Tree \\
\hline \multicolumn{5}{|l|}{ Myristicaceae } \\
\hline Myristica fragrans Houtt & Pala & Liver, nuasea & $\mathrm{BE}, \mathrm{MA}$ & Tree \\
\hline \multicolumn{5}{|l|}{ Nyctaginaceae } \\
\hline Mirabilis jalapa $\mathrm{L}$. & Asari & Poisoning, fever, pimples & $\mathrm{LA}, \mathrm{LO}, \mathrm{BO}$ & Herb \\
\hline \multicolumn{5}{|l|}{ Oxalidaceae } \\
\hline Averrhoa bilimbi $\mathrm{L}$. & Belimbing & Odema & TG, BO & Tree \\
\hline Averrhoa carombola $\mathrm{L}$. & Belerang & Injury & $\mathrm{LO}, \mathrm{BU}$ & Tree \\
\hline Oxalis corniculata $\mathrm{L}$. & Piaewehe & Fever & TG & Shrub \\
\hline \multicolumn{5}{|l|}{ Oleaceae } \\
\hline Jasminum sambac L. & Manuru & Eye infection, face tonic & TG, BE, LO & Shrub \\
\hline
\end{tabular}




\begin{tabular}{|c|c|c|c|c|}
\hline \multicolumn{5}{|l|}{ Pandanaceae } \\
\hline Pandanus udoratissimus L. & Pondang & Cholesterol-lowering, poisoning & TG, LA, BO & Herb \\
\hline \multicolumn{5}{|l|}{ Poaceae } \\
\hline Andropogon nardus Roxb & Watang & Increasing blood circulation & MA, LO & Herb \\
\hline Imperata cylindrical (L.) Raeusch & Hei & Measles, kidney disease & $\mathrm{LO}, \mathrm{BO}$ & Herb \\
\hline Saccharum officinarum L. & Tuwu mahamu & Diabetes mellitus & LA, KE, LO & Herb \\
\hline \multicolumn{5}{|l|}{ Papilionaceae } \\
\hline Crotalaria striata DC & Kuhung-kuhung & Cancer & $\mathrm{BO}$ & Herb \\
\hline Derris elliptica Benth & Tuwa & Poisoning, tumor & NA, LO, BU & Herb \\
\hline Erythrina variegate L. & Bahu & Asthma & $\mathrm{KE}, \mathrm{MA}$ & Tree \\
\hline Sesbania grandiflora $(\mathrm{L})$ Pers & Turi & Sprue & $\mathrm{BU}$ & Shrub \\
\hline \multicolumn{5}{|l|}{ Piperaceae } \\
\hline Piper betle L. & Deling & $\begin{array}{l}\text { Eye infection, fever, internal } \\
\text { disease, baby caring }\end{array}$ & TG, BE, NA, KE, MA, BO & Herb \\
\hline Piper caninum $\mathrm{L}$. & Deling asu & Internal disease & MA & Herb \\
\hline Piper nigrum $\mathrm{L}$. & Rica Jawa & Internal disesae & BU, BO & Herb \\
\hline Peperomia pellucida (L.) Kunth & Rumpu kakidumang & Cholesterol & TG & Herb \\
\hline \multicolumn{5}{|l|}{ Plantaginaceae } \\
\hline Plantago lanceolata $\mathrm{L}$. & Mostor cina & Kidney disease & TG, LO, BO & Herb \\
\hline \multicolumn{5}{|l|}{ Portulacaceae } \\
\hline Talinum triangulare (J. wild) & Ginseng Putih & Aphrodisiac & LO & Herb \\
\hline \multicolumn{5}{|l|}{ Rubiaceae } \\
\hline Gardenia augusta Merr. & Pica piring & Liver & $\mathrm{LA}, \mathrm{LO}, \mathrm{BU}$ & Shrub \\
\hline Morinda citrifolia $\mathrm{L}$. & Seha & Hypertension, liver & TG, LA, KE, MA, LO & Tree \\
\hline Mussaenda pubescens Dryand & Lembawua & Measles, sprue & $\mathrm{TG}, \mathrm{KE}, \mathrm{LO}$ & Shrub \\
\hline \multicolumn{5}{|l|}{ Solanaceae } \\
\hline Capsicum annuum $\mathrm{L}$. & Hisa & Ulcer & NA & Herb \\
\hline Physalis peruviana $\mathrm{L}$. & Letahe & Kidney disease & LA & Herb \\
\hline Solanum lycopersicum $\mathrm{L}$. & Samate & Ulcer & $\mathrm{LO}, \mathrm{BU}$ & Herb \\
\hline Solanum melongena $\mathrm{L}$. & Poki-poki & Dog bite & TG, BE, LA & Shrub \\
\hline Solanum torvum SW & Pumpanikiang & Headache & LO & Shrub \\
\hline \multicolumn{5}{|l|}{ Sterculiaceae } \\
\hline Sterculia sp. & Betadin & Injury & $\mathrm{BU}, \mathrm{BO}$ & Shrub \\
\hline Theobroma cacao $\mathrm{L}$. & Coklat mahamu & Diarrhea, ulcer & $\mathrm{LO}, \mathrm{BO}$ & Tree \\
\hline \multicolumn{5}{|l|}{ Verbenaceae } \\
\hline Clerodendrum calamitosum $\mathrm{L}$. & Gambir & Kidney disease & TG & Tree \\
\hline Clerodendrum sahelanggii Koor Verb & Palata & Fever & $\mathrm{KE}, \mathrm{MA}$ & Shrub \\
\hline Clerodendrum seratum Spreng & Sesewanua & Fever & MA & Shrub \\
\hline Lantana camara $\mathrm{L}$. & Rumput macam & Injury & $\mathrm{LO}, \mathrm{BU}$ & Shrub \\
\hline Strachytarpheta jamaicensis (L.) Vahl. & Kali-kali meo & Kidney disease & $\mathrm{LO}, \mathrm{BU}$ & Herb \\
\hline \multicolumn{5}{|c|}{ Zingiberaceae } \\
\hline Alpinia galanga $(\mathrm{L}$.$) Mild$ & Linkuasi & Scabies & TG, BT, KE, LO & Herb \\
\hline Curcuma longa $\mathrm{L}$. & Kuni & Liver, cough, body odor & $\begin{array}{l}\text { TG, BE, LA, NA, KE, MA, } \\
\text { LO, BU, BO }\end{array}$ & Herb \\
\hline Curcuma xanthorrhiza Roxb. & Timbulawa & Liver & TG, LA, KE, MA, BO & Herb \\
\hline Kaempferia galanga (L.) Milid & Kinsuli & Fever, hypertension, beaty care & TG, LA, KE, MA, BO & Herb \\
\hline Zingiber oficinale Rosc. & Wuho & Cough, headache & $\begin{array}{l}\text { TG, BE, LA, NA, KE, MA, } \\
\text { LO, BU, BO }\end{array}$ & Herb \\
\hline Zingiber purpureum Rosc. & Manggele & Headache & LA & Herb \\
\hline
\end{tabular}

Note: BE: Batulewehe, BO: Bawo, BU: Bawoleu, KE: Kalakube, LA: Lesa, LO: Lumbo, MA: Mala, NA: Naha, TG: Tapuang

\section{Dischidia nythesiana}

Dischidia nythesiana, locally called lintakuhi by the Sanger tribe, is an epiphytic plant belonging to Asclepiadaceae family (Figure 5.A). The drug from this plant is prepared by boiling the tender plants along with the leaves of $D$. pentandra which is a parasite on it, to get a water extract which is consumed orally. There are no scientific reports so far on the medicinal uses and chemical content of D. nythesiana. Utilization of Dischidia imbricata and D. nummularia as a cure for cancer is well known to sellers of herbal medicines in the traditional market of Kabanjahe, North Sumatra (Silalahi et al. 2015b). As there are no published reports on the phytochemicals of $D$. nythesiana, this plant needs to be subjected to phytochemical investigations and cytotoxic tests to validate its anticancerous claim.

\section{Artemisia vulgaris}

Artemisia vulgaris, locally known as tatutup which means 'closed leaves' in local language, is a plant belonging to Asteraceae family (Figure 5.B). Its leaves are squeezed in water to get a liquid extract which is mixed with egg yolk and honey before consumption. Honey is added to reduce bitterness, while egg yolk helps to improve the efficacy of the drug. Patients in early-stage of cancer usually take this preparation once a day while those in advanced stages of cancer are suggested to consume it three times a day. 


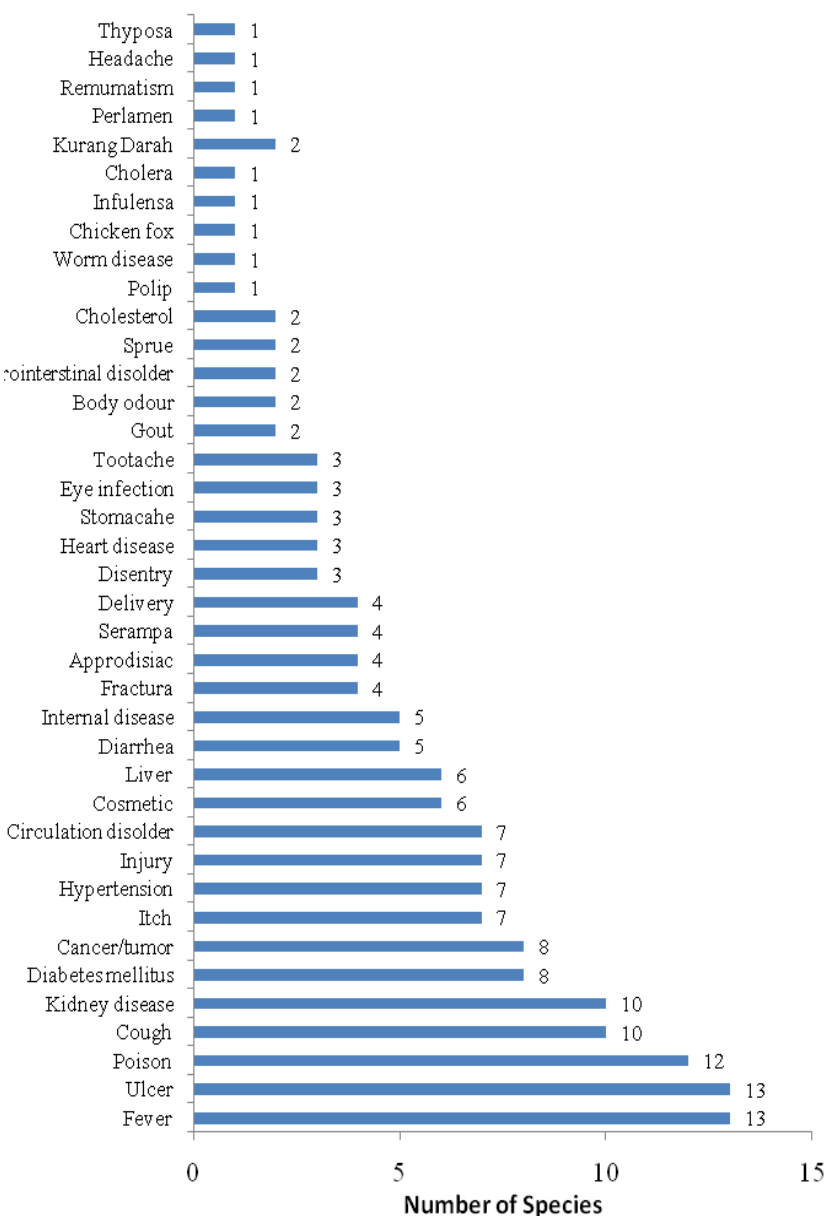

Figure 3. Diseases and number of medicinal plant species used against each disease by the Sanger tribe in the Sangihe Islands, Indonesia

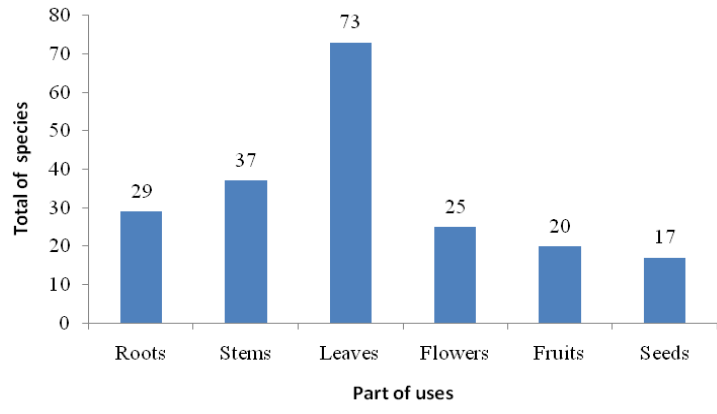

Figure 4. Number of medicinal species based on plant parts used by the Sanger tribe.

The people of Sanger tribe generally prone to smoking habit, which could be the major risk factor for oral cancer. Arundina et al. (2015) reported the potential of $A$. vulgaris as an anti-gastrointestinal cancer drug, especially against oral and breast cancers. Sucipto et al. (2014) listed the main essential oils of $A$. vulgaris, such as 3,5-dimethyl-4ethylidene-cyclohex-2-ena-1-one, filifolone, germacrene$\mathrm{D}$, gamma-caryophyllene, eukarvone and 1,8-cineol, each with a level greater than $3 \%$. The anticancer property of $A$. vulgaris is suspected to be associated with its essential oil content. The action of essential oils as anti-cancer agents has been reported by Zarlaha et al. (2014), who extracted the oil of Ocimum basilicum and tested its anti-cancer activity in different human cell cancer cells, namely HeLa cells, adenocarcinoma cells, cervical cancer cells, FemX human melanoma cells, K562 chronic myelogenous leukemia cells and SKOV3 human ovarian cells, through in vitro experiments.

\section{Crotalaria striata}

Crotalaria striata or kuhung-kuhung in local language is a member of Fabaceae family (Figure 5.C). Sanger people consume it as a water extract obtained by boiling the whole plants. Nurrani et al. (2016) reported the potential of ethanol extract of $C$. striata leaves as an anticervical cancer drug. Moreover, the ethyl acetate fraction of Crotalaria sp. extract was found to inhibit MCF-7 breast cancer cells with the lowest IC50 value of $29.67 \mu \mathrm{g} / \mathrm{mL}$, followed by the n-hexane and ethyl acetate fractions of the seed extract and the ethanol fraction of the leaf extract with IC50 values of 32.1, 44.2and $56.7 \mu \mathrm{g} / \mathrm{mL}$, respectively. The hexane and ethyl acetate fractions of the leaf and seed extracts had IC50 value of 107.14 and170.83 $\mu \mathrm{g} / \mathrm{mL}$, respectively, and they are effective against the MCF-7 breast cancer cells. However, the ethanol extract of the seeds and leaves did not show inhibition of proliferation of MCF-7 breast cancer cells (Rumondor et al. 2016).

\section{Eleutherine americana}

Eleutherine americana is called bawang babi in Sanger dialect and bawang Dayak in Bahasa, Indonesia (Figure 5.D) due to its medicinal use among the Dayak ethnic group (Kuntorini and Nugroho 2010). Moreover, $E$. americana is also in use as a spice for cooking purposes among the local communities of Asia, including the Dayak tribe. It may be assumed that utilization of E. americana by the Sanger tribe to cure cancer was adopted from the local wisdom of the Dayak tribe who use it as a breast cancer drug. This phenomenon shows the acculturation of local wisdom from different tribes (Sujarwo and Caneva 2016). To use it as a medicine, the plant is prepared by boiling the tuberous bulbs and the water extract obtained is filtered prior to consumption.

Eleutherine americana has been widely reported as an anti-cancer drug especially for breast cancer (Kuntorini and Nugroho 2010; Saragih et al. 2014; Amelia et al. 2015). Several studies have reported that the bulb of E. americana contain phytochemicals such as naphthalene, anthraquinone and naphthoquinone (Insanu et al. 2014); elecanacine, eleutherine, eleutherol, and eleuthernone (Hara et al. 1997); eleutherol, eleutherinoside $\mathrm{A}$ and $\mathrm{B}$, and the potentiallymedicinal eleuthoside (Ieyama et al. 2011); and 6,8dihydroxy- 3,4-dimethoxy- 1-methyl-anthraquin-one-2carboxylic acid methyl ester (Xu et al. 2006). Eleutherine, eleutherinone, eleutherol, isoeleutherine, eleutherinol, dihydroeleutherinol, hongconin and 6,8-dihydroxy-3,4dimethoxy- 1-methyl-anthraquinone- 2-carboxylic acid methyl esters have been shown to inhibit the growth of HeLa cells (Amelia et al. 2015). The compound 6,8dihydroxy- 3,4-dimethoxy- 1-methyl-anthraquin-one-2- 
carboxylic acid methyl ester was also reported to have the ability to inhibit erythroleukemia cell line K562 with IC50 value of $49.1 \mu \mathrm{g} / \mathrm{mL}$ (Xu et al. 2006).

\section{Diversity of medicinal plants across study villages}

The Sanger tribe utilizes as many as 118 species of medicinal plants. The number of medicinal plants utilized by the Sanger tribe in different villages selected for the study varied from the lowest of 27 species, which was recorded from Mala Village, to the highest of 54 species recorded from Lumbo Village (Figure 6). This variation is influenced mainly by the diversity of plants found in the surrounding environment, the topography of the village area and its distance to the forests. A total of 7 species were found to be commonly used as medicinal in all nine research sites. They are J. curcas, Abelmoschus manihot, Psidium guajava, Allium schoenoprasum, Persea americana, $C$. domestica, and $Z$. officinale. These plants are usually cultivated and commonly found in various regions of Indonesia. In addition to medicinal purposes, some of them are also used for other purposes, such as edible fruits ( $P$. guajava, $P$. americana), kitchen spices $(Z$. officinale, A. schoenoprasum), and as hedgerow plant ( $J$. curcas)

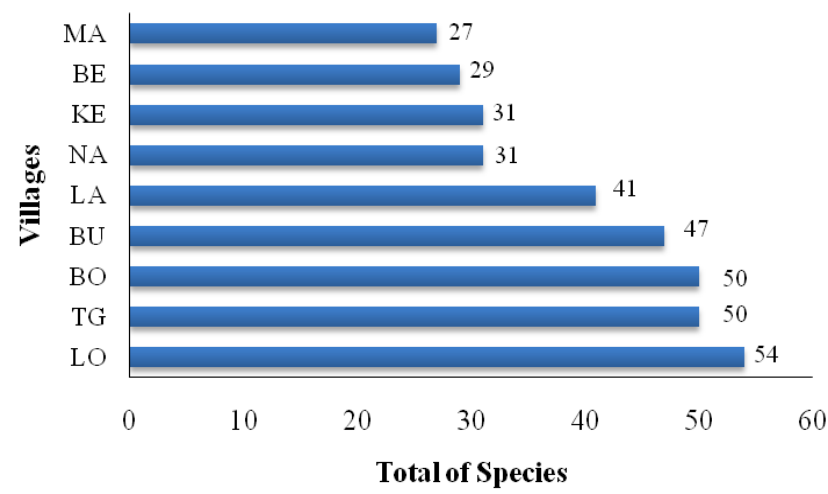

Figure 6. The number of medicinal plant species utilized by the Sanger tribe in different study villages. BE: Batulewehe, BO: Bawo, BU: Bawoleu, KE: Kalakube, LA: Lesa, LO: Lumbo, MA: Mala, NA: Naha, TG: Tapuang

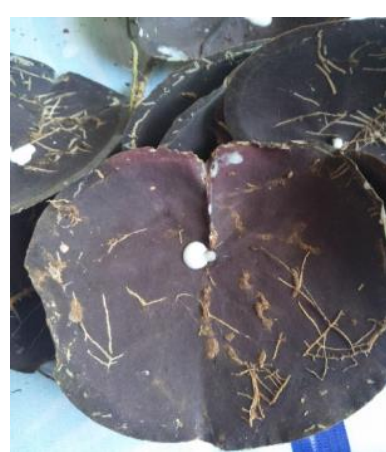

A

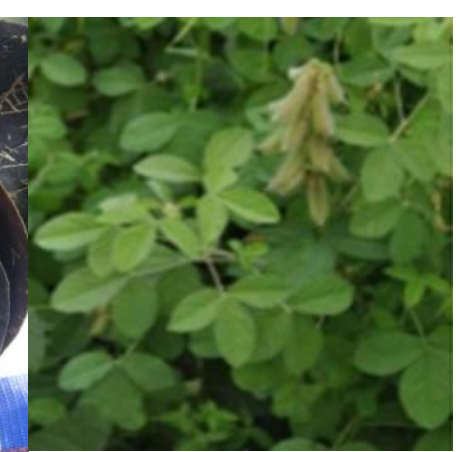

B

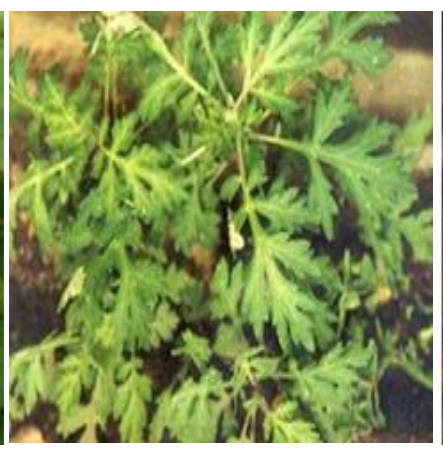

C

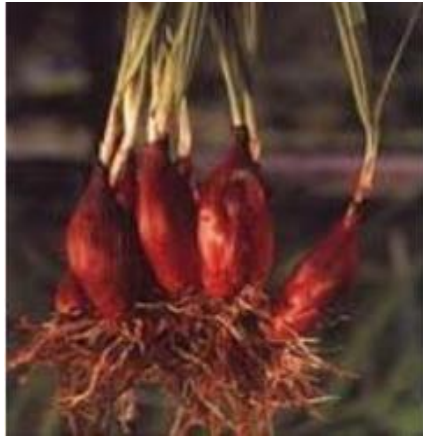

D

Figure 5. Traditional anti-cancer medicinal plants used by the Sanger tribe in the Sangihe Islands: A. Dischidia nythesiana, B. Crotalaria striata, C. Artemisia vulgaris, and D. Eleutherine americana.

Table 2. The Jaccard similarity index of the medicinal plants used in different villages by the Sanger tribe in Sangihe Islands, Indonesia

\begin{tabular}{lccccccccc}
\hline & TG & BU & BE & BO & LA & LO & NA & KE & MA \\
\hline TG & - & 0.260 & 0.317 & 0.400 & 0.371 & 0.250 & 0.286 & 0.350 & 0.242 \\
BU & 0.26 & - & 0.394 & 0.342 & 0.236 & 0.500 & 0.262 & 0.242 & 0.189 \\
BE & 0.317 & 0.394 & - & 0.254 & 0.333 & 0.258 & 0.200 & 0.224 & 0.273 \\
BO & 0.400 & 0.342 & 0.254 & - & 0.333 & 0.333 & 0.270 & 0.246 & 0.262 \\
LA & 0.371 & 0.236 & 0.333 & 0.333 & - & 0.263 & 0.296 & 0.327 & 0.308 \\
LO & 0.250 & 0.500 & 0.258 & 0.333 & 0.263 & - & 0.250 & 0.219 & 0.227 \\
NA & 0.286 & 0.262 & 0.200 & 0.270 & 0.296 & 0.250 & - & 0.292 & 0.289 \\
KE & 0.35 & 0.242 & 0.224 & 0.246 & 0.327 & 0.219 & 0.292 & - & 0.415 \\
MA & 0.242 & 0.189 & 0.273 & 0.262 & 0.308 & 0.227 & 0.289 & 0.415 & - \\
\hline
\end{tabular}

Note: BE: Batulewehe, BO: Bawo, BU: Bawoleu, KE: Kalakube, LA: Lesa, LO: Lumbo, MA: Mala, NA: Naha, TG: Tapuang 
The medicinal plants used by the Sanger tribe are different across studied villages and some species are specifically used only in certain villages. For example, Peperomia pellucida was used only in Tapuang Village, Talinum triangulare only in Lumbo Village, Alstonia scholaris in Bawo, Homalomena occulta in Bawoleu, D. nythesiana in Lumbo and Bawo, and Spilathes ocymifolia in Lumbo. The calculated Jaccard similarity indices were between 0.189 and 0.500 (Table 2). This shows that the medicinal plants used by each village were relatively different from those of other villages. The lowest similarity index of 0.189 existed between Mala (MA) and Bawoleu (BU) villages, whereas the highest similarity index of 0.500 existed between Bawoleu (BU) and Lumbo (LO) villages. Silalahi and Nisyawati (2018) found that in the Karo ethnic group, adjacent villages had higher Jaccard equality indexes than those located away from each other. Communities in neighboring villages have a wider opportunity to exchange information on the use of medicinal plants compared to more distant villages.

The Lumbo village did not have any health service facility, so the villagers used mainly traditional medicine for maintaining their health. Therefore, many medicinal plants were found in cultivation in this village. Moreover, the Lumbo village is relatively close to the forest, compared to other villages. Mala village was found to be using the lowest number of medicinal plants (27 species), as this village is near to urban areas and it has proper health care facilities. Furthermore, the knowledge of medicinal plants among the villagers in Mala was found to be lower compared to that of the Lumbo village and they cultivated less than number of medicinal plants.

\section{ACKNOWLEDGEMENTS}

The authors wish to express their gratitude to sister Endang Mulalinda and family who helped to retrieve the data and confirm the previous data. They also thank the Indonesian Government for research grant through the Higher Education Research Grant in 2015 and through UNSRAT PNBP funds in 2017.

\section{REFERENCES}

Achan J, Talisuna AO, Erhart A, Yeka A, Tibenderana JK, Baliraine FN, Rosenthal PJ, D'Alessandro U. 2011. Quinine, an old anti-malaria drug in a modern world: role in the treatment of malaria. Malar J 10: 144. DOI: $10.1186 / 1475-2875-10-144$.

Alexandrova R, Alexandrova I, Velcheva M, Varadinova T. 2000. Phytoproduct and Cancer: Experimental Pathology and Parasitology. Bulgarian Academy of Sciences, Sophia.

Alexiades MN, Sheldon JW. 1996. Selected Guidelines for Ethnobotanical Research: A Field Manual. New York Botanical Garden Press, New York.

Amelia T, Pratiwi D, Romsiah, Tjahjono DH. 2015. In silico study of the component of Eleutherine americana Merr. on human estrogen receptor alpha as potential anti-breast cancer. The 3rd International Conference on Computation for Science and Technology (ICCST-3), Bali-Indonesia.

Anwar J, Damanik SJ, Hisyam N, Whitten AJ. 1984. Ekologi Ekosistem Sumatera, Gajah Mada University Press, Yogyakarta. [Indonesian]
Arundina I, Budhy STI, Luthfi M, Indrawati R. 2015. Identification of Sudamala (Artemisia vulgaris L.) Thin Layer Chromatography. Majalah Kedokteran Gigi Indonesia 1 (2): 167-171. DOI: 10.22146/majkedgiind.9226

Backer CA, Bakhuizen v.d. Brink Jr RC. 1963. Flora of Java. Vol. I. P. Noordhoff, Groningen.

Backer CA, Bakhuizen v.d. Brink Jr RC. 1965. Flora of Java. Vol. II. P. Noordhoff, Gronigen.

Backer CA, Bakhuizen v.d. Brink Jr RC. 1968. Flora of Java. Vol. III. P. Noordhoff, Gronigen.

BPS. 2017. Kabupaten Kepulauan Sangihe Dalam Angka 2017. Badan Pusat Statistik Kabupaten Kepulauan Sangihe, Sangihe. [Indonesian]

BPS. 2018. Kabupaten Kepulauan Siau Tagulandang Biaro Dalam Angka 2018. Badan Pusat Statistik Kabupaten Kepulauan Siau Tagulandang Biaro, Sitaro. [Indonesian]

Bulut W. 2005. Inventarisasi Tumbuhan Obat di Kecamatan Passi, Modayag, Lolayan Kabupaten Bolaang Mongondow. [Hon. Thesis]. FMIPA, Universitas Sam Ratulangi, Manado.

Cox CB, Moore PD. 2008. Biogeography. An Ecological and Evolutionary Approach. 7th ed. Blackwell Publishing, New York.

Fabricant DS, Farnsworth NR. 2001. The value of plant used medicine for drug discovery. Environ Health Perspect 109 (1): 69-75.

Hara H, Maruyama N, Yamashita S, Hayashi Y, Lee KH, Bastow KF, Chairul, Marumoto R, Imakura Y. 1997. Elecanacin, a novel new naphthoquinone from the bulb of Eleutherine americana. Chem Pharm Bull 45: 1714-1716.

Harmida S, Yuni VF. 2010. Studi etnofitomedika di desa Lawang Agung Kecamatan Mulak Ulu Kabupaten Lahat Sumatera Selatan. Jurnal Penelitian Sains 14 (1): 42-46. [Indonesian]

Hoang VS, Bas P, Kebler PAJ. 2008. Traditional medicine plant in Ben En National Park, Vietnam. Blumea 53: 569-601.

Ieyama DT, Maria DPT, Puteri G, Kawabata J. 2011. a-Glucosidase inhibitors from the bulb of Eleutherine americana. Food Chem 128: 308-311.

Indrawan M, Primack RB, Supriatna J. 2007. Biologi Konservasi (Edisi Revisi). Yayasan Obor Indonesia. Jakarta. [Indonesian]

Insanu M, Kusmardiyani S, Hartati R. 2014. Recent studies on phytochemicals and pharmacological effects of Eleutherine americana Merr. Procedia Chem 13: 221-228.

Kandou FE, Pandiangan D. 2006. Inventarisasi dan penapisan alkaloid tumbuhan obat tradisional suku Sanger, Kabupaten Sangihe Sulawesi Utara. Eugenia 12 (2): 95-101. [Indonesian]

Kartawinata K. 2010. Dua abad mengungkap kekayaan floradan ekosistem Indonesia. Dalam: Sarwono Prawirohardjo Memorial Lecture X. LIPI. 23 Agustus 2010, Jakarta. [Indonesian]

Khairiah A. 2017. Etnomedisin dan Nilai Ekonomi Tumbuhan Obat Pada Etnis Minangkabau di Kecamatan IX Koto Sungai Lasi, Solok, Sumatra Barat. [Thesis]. Departemen Biologi, FMIPA, Universitas Indonesia, Depok. [Indonesian]

Krebs CJ. 1978. Ecological Methodology. Harper and Row Publisher, New York.

Kuntorini EM, Nugroho LH. 2010. Structural development and bioactive content of red bulb plant (Eleutherine americana); a traditional medicine for local Kalimantan people. Biodiversitas 11 (2): 102-106.

Martin GJ. 1995. Ethnobotany a People and Plants Conservation Manual. Chapman and Hall. London, UK.

MoH. 1977. Materia Medika Indonesia, Jilid I. Departemen Kesehatan, Republik Indonesia, Jakarta. [Indonesian]

Mulalinda E, Pandiangan D, Dapas F. 2006. Inventarisasi tumbuhan obat tradisional di Kepulauan Sangihe. [Hon. Thesis]. FMIPA, Universitas Sam Ratulangi, Manado. [Indonesian]

Müller-Dombois D, Ellenberg H. 1974. Aims and Methods of Vegetation Ecology. John Wiley and Sons, New York.

Munim A, Hanani E. 2011. Fisioterapi Dasar. Dian Rakyat, Jakarta. [Indonesian]

Nawangningrum D, Widodo S, Suparta IM, Holil M. 2004. Kajian terhadap naskah kuna Nusantara koleksi Fakultas Ilmu Pengetahuan Budaya Universitas Indonesia: penyakit dan pengobatan ramuan tradisional. Makara Sosial Humaniora 8 (2): 45-53. [Indonesian]

Nurrani L, Tabba S, Irawan A. 2016. Bioactivity of Crotalaria striata Dc and Cinnamomum cullilawan $\mathrm{Bl}$ against cervical cancer cells of HeLa. Bul Littro 27 (1): 1-9.

Pandiangan D, Kandou FE. 2006. Inventarisasi dan penapisan alkaloid tumbuhan obat tradisional Suku Sanger di Sangihe. Seminar Nasional Farmasi, Universitas Padjadjaran, Bandung. [Indonesian] 
Pangemanan LD, Rondonuwu SJ. 1991. Bioekologi dan Inventarisasi Penggunaan Tumbuhan Tradisional di Kabupaten Bolang Mongondow. Universitas Sam Ratulangi, Manado.

Purba EC, Nisyawati, Silalahi M. 2016. The ethnomedicine of the Batak Karo Peoples of Merdeka Subdistrict, North Sumatra, Indonesia. Intl J Biol Res 4 (2): 181-189

Purwanto Y. 2002. Studi etnomedisinal dan fitofarmakope tradisional Indonesia. Prosiding Seminar Nasional II Tumbuhan Obat dan Aromatik. LIPI, Bogor. [Indonesian]

Rumondor EM, Moelyono MW, Barliana MI. 2016. Aktivitas antiproliferasi ekstrak dan fraksi daun kuhung-kuhung (Crotalaria pallida Aiton) terhadap Sel kanker payudara MCF-7. Jurnal Farmaka 14 (2): -. [Indonesian]

Saragih B, Pasiakan M, Saraheni, Wahyudi. 2014. Effect of herbal drink plants Tiwai (Eleutherine americana Merr) on lipid profile of hypercholesterolemia patients. Intl Food Res J 21 (3): 1199-1203.

Silalahi M, Nisyawati, Walujo EB, Supriatna J, Mangunwardoyo W. 2015a. The local knowledge of medicinal plants trader and diversity of medicinal plants in the Kabanjahe traditional market, North Sumatra, Indonesia. J Ethnopharmacol 175: 432-443.

Silalahi M, Nisyawati, Walujo EB, Supriatna J. 2015b. Local knowledge of medicinal plants in sub-ethnic Batak Simalungun of North Sumatra, Indonesia, Biodiversitas 16 (1): 44-54

Silalahi M, Nisyawati. 2018. The ethnobotanical study of edible and medicinal plants in the home garden of Batak Karo sub-ethnic in North Sumatra, Indonesia. Biodiversitas 19 (1): 229-238.

Simbala H, Potabuga I. 2002. Tumbuhan Obat Masyarakat Suku Bogani Kabupaten Mongondow Sulawesi Utara. FMIPA, Universitas Sam Ratulangi, Manado. [Indonesian]

Soetjipto H, Elok EB, Linawati L. 2014. Pengaruh berbagai metoda penyulingan terhadap komponen penyusun minyak atsiri tanaman baru Cina (Artemisia vulgaris L) serta efek antibakterinya. Jurnal Penelitian Saintek 19 (2): -. [Indonesian]

Sujarwo W, Caneva G. 2016. Using quantitative indices to evaluate the cultural importance of food and nutraceutical plants: Comparative data from the Island of Bali (Indonesia). J Cult Herit 18: 342-348.
ThePlantlist. 2017. The Plantlist Database. www.theplantlist.org. [2 October 2017]

Wahyono. 2008. Eksistensi dan perkembangan obat tradisional (jamu) dalam era obat modern. Fakultas Farmasi, Universitas Gajah Mada, Yogyakarta. [Indonesian]

Wardah, Danimiharja S. 1996. Pemanfaatan tumbuhan sebagai obat tradisional berbagai daerah kawasan Taman Nasional Dumogo Bone, Sulawesi Utara. Prosiding Simposium Nasional Penelitian Bahan Obat Alami VIII. Balitbang Botani, Puslitbang Biologi, LIPI, Bogor. [Indonesian]

Widadeti, Roemantyo. 1996. Pemanfaatan tumbuhan untuk pengobatan tradisional penyakit rakyat di Dumogo Bone Sulawesi Utara. Prosiding Seminar dan Loka Karya Nasional Etnobotani II. Departemen Pendidikan dan Kebudayaan RI \& LIPI, Bogor. [Indonesian]

Wijayakusuma H, Dalimartha S, Wirian A. 1996a. Tanaman berkhasiat obat di Indonesia. Jilid 1. Pustaka Kartini Jakarta. [Indonesian]

Wijayakusuma H, Dalimartha S, Wirian A. 1996b. Tanaman berkhasiat obat di Indonesia. Jilid 3. Pustaka Kartini Jakarta. [Indonesian]

Wijayakusuma H, Dalimartha S, Wirian A. 1996c. Tanaman berkhasiat obat di Indonesia. Jilid 4. Pustaka Kartini, Jakarta. [Indonesian]

Wijayakusuma H. 2000. Ensiklopedia Milenium Tumbuhan Berkhasiat Obat Indonesia. Prestasi Insan Indonesia. Jakarta. [Indonesian]

Windardi FI, Uji T. 1996. Pemanfaatan Tumbuhan dalam Pengobatan Tradisional: Studi Kasus Masyarakat Pedesaan di Desa Pindol dan Totabuan Kecamatan Lolak Kabupatenn Bolaan Mongondow Sulawesi Utara. Prosiding Simposium Nasional Tumbuhan Nasional Tumbuhan Obat dan Aromatik Obat Alami VIII. Balitbang Botani, Puslitbang Biologi LIPI, Bogor. [Indonesian]

Xu JZ, Qiu F, Duan WJ, Qu GX, Wang N, Yao XS. 2006. New bioactive constituents from Eleutherine americana. Front Chem China 1 (3): 320-323.

Zarlaha A, Kourkoumelis N, Stanojkovic TP, Kovala-Demertzi D. 2014. Cytotoxic activity of essential oil and extracts of Ocimum basilicum against human carcinoma cells. Molecular docking study of isoeugenol as a potent cox and lox inhibitor. Digest $\mathbf{J}$ Nanomater Biostruct 9 (3): 907-917. 